\title{
Systematic Endoscopic Approach to Early Gastric Cancer in Clinical Practice
}

\author{
Gwang Ha Kim \\ Department of Internal Medicine, Pusan National University College of Medicine, and Biomedical Research Institute, Pusan National \\ University Hospital, Busan, Korea
}

Article Info
Received October 23, 2020
Revised December 9, 2020
Accepted December 28, 2020
Corresponding Author
Gwang Ha Kim
ORCID https://orcid.org/0000-0001-9721-5734
E-mail doc0224@pusan.ac.kr

E-mail doc0224@pusan.ac.kr

\begin{abstract}
Early gastric cancers (EGCs) are defined as gastric cancers confined to the mucosa or submucosa, regardless of regional lymph node metastasis. The proportion of EGCs has been increasing due to the increase in screening endoscopy for gastric cancers; therefore, the paradigm shift from surgical resection to endoscopic resection as a treatment modality for selected EGCs is accelerating. For successful endoscopic resection of EGCs, it is important to detect EGCs at an early stage and to accurately predict the histological type, depth of invasion, and horizontal margins of the tumor. The diagnostic process of EGCs can be divided into three steps: presence diagnosis, qualitative diagnosis, and quantitative diagnosis. The presence diagnosis of EGCs is mainly based on two endoscopic findings: a well-demarcated lesion and irregularity in the color/surface pattern. Qualitative diagnosis refers to the prediction of histological type, which is mainly possible based on the macroscopic shape and color of the lesion. Quantitative diagnosis of EGCs consists of predicting the depth of invasion by detailed examination of the macroscopic morphology and determining horizontal margins using chromoendoscopy. Although advanced diagnostic modalities, such as endosonography or magnifying endoscopy, are helpful for the qualitative and quantitative diagnosis of EGCs, these modalities are not available in most hospitals. Therefore, it is still very important to evaluate EGCs systematically during conventional endoscopy for successful endoscopic treatment. (Gut Liver 2021;15:811-817)
\end{abstract}

Key Words: Stomach neoplasms; Endoscopy; Diagnosis

\section{INTRODUCTION}

Early gastric cancers (EGCs) are defined as gastric cancers confined to the mucosa or submucosa, regardless of regional lymph node metastasis. The proportion of EGCs among all gastric cancers has been increasing steadily because of the increase in screening endoscopy for gastric cancers, especially during the national gastric cancer screening programs in Korea and Japan. ${ }^{1,2}$ As a result, the paradigm away from surgical resection to endoscopic resection as a treatment modality for selected EGCs is accelerating.

The standard indications for endoscopic mucosal resection (EMR) of EGCs are differentiated-type, mucosal, elevated cancers $\leq 20 \mathrm{~mm}$ in size; or depressed cancers $\leq 10$ $\mathrm{mm}$ in size, without ulceration or lymph node metastasis. ${ }^{3}$
However, EMR is not recommended for EGCs $>20 \mathrm{~mm}$ in size or those with ulceration because of piecemeal resection and local tumor recurrence. ${ }^{4}$ Endoscopic submucosal resection (ESD) is the most advanced and preferred technique for treating EGCs, with specialized devices, such as an insulation-tipped knife and electrosurgical unit. ESD enables the resection of large or ulcerated lesions en bloc, which reduces the recurrence rate compared to EMR. Therefore, the expanded criteria for endoscopic resection of EGCs that are commonly used in many countries including the following: (1) differentiated-type mucosal cancers without ulceration, regardless of the tumor size; (2) differentiated-type mucosal cancers $\leq 3 \mathrm{~cm}$ in size with ulceration; (3) differentiated-type minute submucosal invasive cancer $\leq 3 \mathrm{~cm}$ in size; and (4) undifferentiated-type mucosal cancer $\leq 2 \mathrm{~cm}$ in size without ulceration. ${ }^{7}$ 

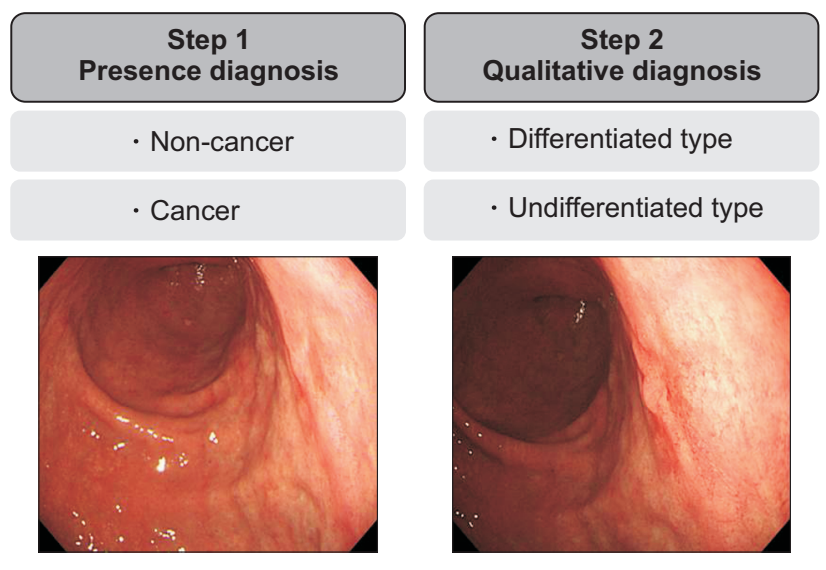

For successful ESD of EGCs based on the abovementioned criteria, it is important to detect EGCs at an early stage and to accurately predict the histological type, depth of invasion, and horizontal margins of the tumor. This review aimed to introduce a systematic approach for EGCs during conventional endoscopy, including the following three steps: (1) presence diagnosis, (2) qualitative diagnosis, and (3) quantitative diagnosis (Fig. 1).

\section{PRESENCE DIAGNOSIS OF EGC}

Marked improvement in endoscopic imaging technologies, such as high-definition videoendoscopes, facilitate the detection of EGCs. However, in order to increase the detection of such lesions, endoscopic maneuvers, such as washing off the adherent mucus by water injection, aspirating the remaining gastric juice, and controlling the amount of air insufflation, are mandatory. The presence diagnosis of EGCs is mainly based on the following two endoscopic findings: a well-demarcated lesion and irregularity in color/surface pattern. ${ }^{8}$

EGCs are macroscopically classified as type 0 as an extension of the Borrmann type, which is further sub-classified into types 0-I to 0-III (Fig. 2). Type 0-I is a protruded type, type $0-\mathrm{II}$ is a superficial type, and type $0-\mathrm{III}$ is an excavated type. Type 0 -II is divided into three types: 0 -IIa (slightly elevated type), 0-IIb (flat type), and 0-IIc (slightly depressed type). Type 0-IIc is the most common type of EGCs. EGCs often appear as a mixture of types, in which case the major type is followed by the minor type (e.g., 0-IIb+IIa, 0-IIc+IIa). In 2003, the Paris classification was established for macroscopic classification of superficial lesions in the gastrointestinal tract. ${ }^{9}$ The difference between type 0-I and type 0-IIa is based on the tumor height of 2.5 $\mathrm{mm}$, and the distinction between $\mathrm{sm} 1$ and $\mathrm{sm} 2$ is based on the depth of submucosal invasion $(500 \mu \mathrm{m}) .^{10}$ The tips for

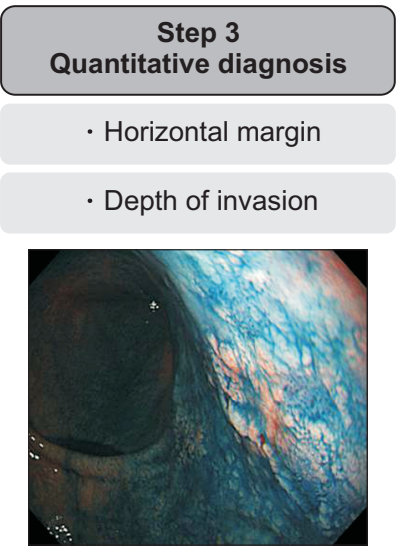

Fig. 1. Schema of systematic endoscopic approach to diagnose early gastric cancer.

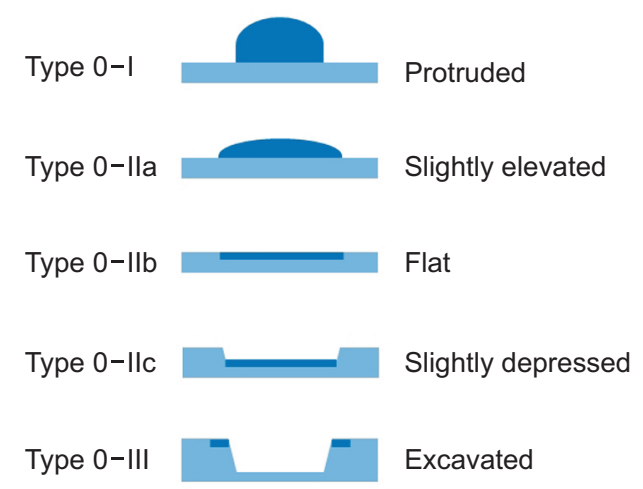

Fig. 2. Macroscopic classification of early gastric cancer. The dark blue color indicates the area occupied by carcinomatous cells. ${ }^{10}$

the detection of EGCs will be described according to the macroscopic shape.

\section{Elevated-type EGCs}

Elevated-type EGCs are relatively easy to diagnose because they are easily noticeable during endoscopy. However, small granular or superficial elevated lesions are difficult to diagnose unless the endoscopists observe carefully. Type 0-I EGCs appear as semi-pedunculated or sessile lesions; they are larger in size than benign polyps and show granular or lobulated shape with a rough surface, changes in color, depression, erosion, ulceration, or easy bleeding tendency. In addition, they are easy to diagnose, as they are often accompanied by 0 -IIa lesions. Although the prevalence of type 0-I EGCs is low, it is difficult to distinguish them from hyperplastic polyps. In general, it is highly likely that a polyp $>2 \mathrm{~cm}$ in size will be cancerous, but even at a smaller size, it is desirable to perform polypectomy to examine the entire tissue if possible.

Type 0-IIa EGCs appear as slightly elevated lesions similar to flat adenomas, making it difficult to distinguish between the two lesions. Type 0-IIa EGCs have erosion, 
bleeding, exudate, irregular unevenness, or color change on their surface. Type 0-IIa+IIc EGCs are difficult to differentiate from raised erosions. Raised erosions are usually multiple, symmetrical, and located at the center of mucosal folds and have inflammatory changes at the center of the erosion, while type 0-IIa+IIc EGCs are single, have an irregular moth-eaten appearance, and are skewed to the side of mucosal folds.

\section{Flat-type EGCs}

Type 0-IIb EGCs are difficult to detect by conventional endoscopy because there is no difference in the height from the surrounding normal mucosa. They can be suspected when there is a change in color, such as discoloration or redness, loss of luster, change in the microsurface structure, or a tendency to bleed easily at the time of air inflation and contract. However, these findings are also commonly observed in atrophic gastritis; therefore, they are not typical characteristics of type 0-IIb EGCs. In general, type 0-IIb EGCs are smaller and have more irregular microsurface structures than chronic inflammation, and they are usually accompanied by type 0 -IIc lesions.

On the basis of color, type 0-IIb EGCs can present as reddish, discolored, or normal in color. ${ }^{11}$ Reddish lesions are most commonly observed, easily detectable, and usually found in the gastric antrum. Discolored lesions are commonly found in the gastric body. Lesions with a normal color can be observed as those with a slightly irregular surface and are very difficult to detect; therefore, they are often diagnosed by incidental biopsy. The size of pure type 0 -IIb EGCs is usually $\leq 2 \mathrm{~cm}$, but that of type 0-IIb EGCs combined with other types is relatively large $(2-6 \mathrm{~cm})$, making it difficult to recognize the exact horizontal extent of the lesion.

\section{Depressed-type EGCs}

Depressed-type EGCs account for $70 \%$ to $80 \%$ of all EGCs and are the most important lesions in the diagnosis of EGCs, as they must be distinguished from various lesions, such as erosions, benign ulcers, and advanced gastric cancers. Type 0-IIc EGCs have definite step-down depression, irregular unevenness, and reddish or pale color. In cases of type 0-IIc EGCs with scar, the distribution of regenerative epithelium is uneven and the margins have a moth-eaten appearance. In cases of EGCs with convergence of mucosal folds, characteristic fold changes, such as abrupt cutting, thinning, clubbing, fusion, or encroachment, are observed, which can be distinguished from benign ulcers in the healing or scar stage.

In type 0-III EGCs, a definite ulceration exists and the carcinoma cells are present only in a narrow portion of the ulcer margins; therefore, it is difficult to differentiate these from benign ulcers in the active or healing stage. They can be differentiated from benign ulcers by the follows points: the shape of the ulcer is irregular, the ulcer base is higher than the surrounding mucosa, it has granular or nodular unevenness, island-like mucosa is observed in the ulcer base, the white exudate is unevenly covered and dirty, hemorrhage or blood clots are often coated, the distribution of regenerative epithelium is uneven, the ulcer margin is hard, variation of converged mucosal folds is severe, and extension of the mucosal folds does not meet at one point.

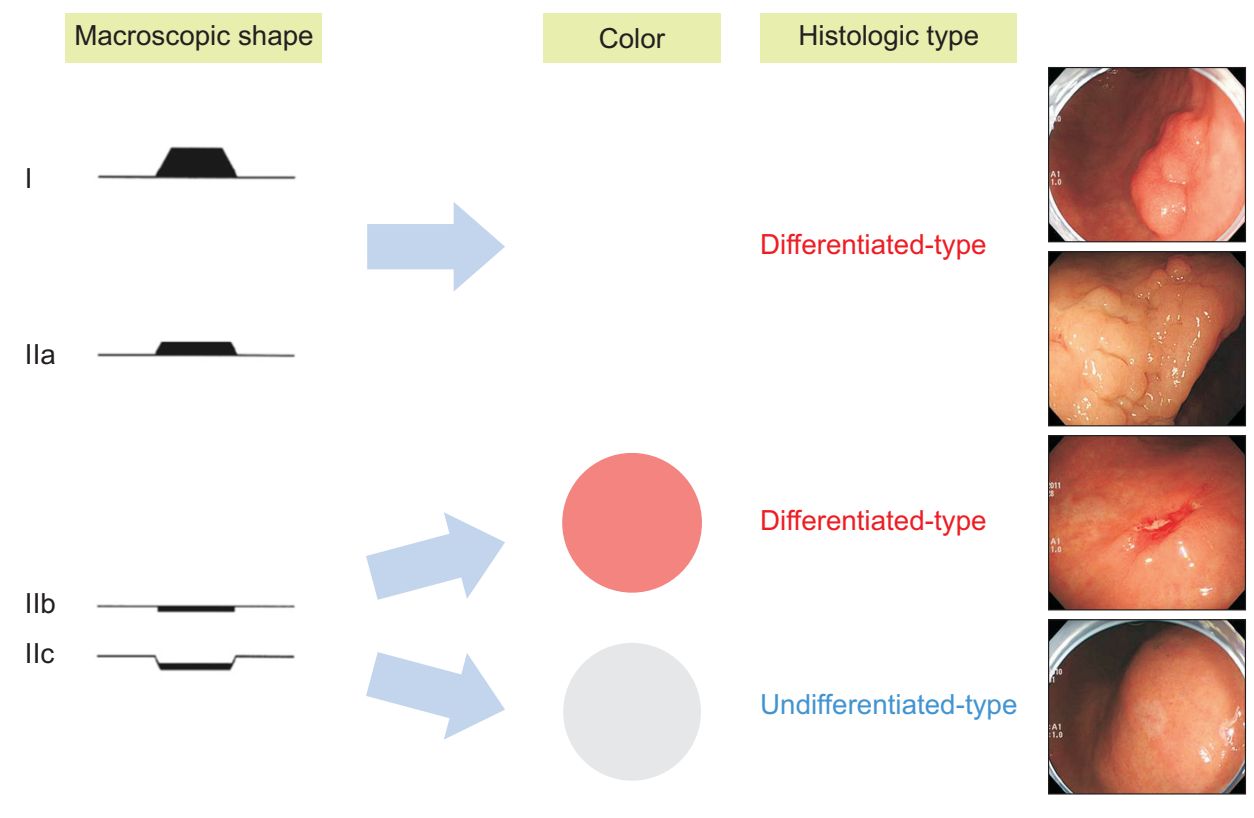

Fig. 3. Endoscopic tips for predicting the histological type of early gastric cancer. 


\section{QULATATIVE DIAGNOSIS OF EGC}

\section{Prediction of histological type}

Usually, the diagnosis of the histological type of EGCs is based on the endoscopic forceps biopsy results. However, as endoscopic biopsy often cannot correctly reflect the final histopathology, ${ }^{12}$ histological discrepancy may occur between endoscopic biopsy and final specimens from endoscopic or surgical resection. ${ }^{13}$ In this situation, endoscopic findings, such as macroscopic shape and color, can provide a useful clue for the histological type of EGCs.

First, the macroscopic shape can help predict the histological type; elevated-type (I, IIa) EGCs are usually differentiated-type cancers, whereas undifferentiatedtype cancers tend to have flat (IIb) or depressed (IIc, III) shape. Next, the color change enables the endoscopists to detect small, flat, or depressed lesions and predict the histological type of gastric cancers; therefore, the color of a lesion observed during endoscopy is important for endoscopic diagnosis of EGCs. ${ }^{14}$ EGCs are observed as reddish or discolored lesions compared to the surrounding non-cancerous mucosa. Histopathological studies have reported that the color change observed during endoscopy in EGCs is associated with the vascularity within the car- cinomatous mucosa; ${ }^{15,16}$ the mucosal vascularity of most differentiated-type cancers is similar or higher compared to the surrounding non-cancerous mucosa, and that of most undifferentiated-type cancers is lower than that of the surrounding non-cancerous mucosa. Therefore, in general, differentiated-type EGCs have the same or reddish color as the surrounding non-cancerous mucosa, whereas undifferentiated-type EGCs are observed as discolored lesions compared to the surrounding mucosa. ${ }^{14,17}$ In summary, endoscopists can predict the histological type of EGCs based on the macroscopic shape and color of the lesions (Fig. 3).

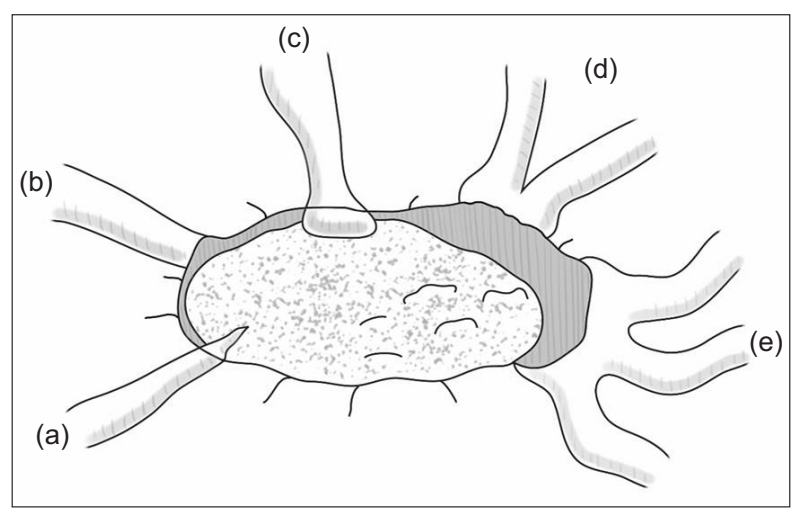

Fig. 4. Morphological changes in the tip of folds in depressed-type cancers. (a) Tapering. (b) Abrupt cutting. (c) Clubbing. (d) Fusion. (e) Bank-like elevation. (a) and (b) suggest mucosal cancer, (c) and (d) suggest submucosal cancer, and (e) suggests advanced cancer.

Table 1. Differentiation Points to Distinguish between Mucosal Cancer and Submucosal Cancer during Endoscopy

\begin{tabular}{|c|c|c|}
\hline Macroscopic shape & Suggesting mucosal cancer & Suggesting submucosal cancer \\
\hline I & $\begin{array}{l}\cdot \leq 2 \mathrm{~cm} \\
\cdot \text { Pedunculated }\end{array}$ & $\begin{array}{l}>2 \mathrm{~cm} \\
\text { Sessile } \\
\text { Uneven surface with nodules } \\
\text { Deep depression } \\
\text { Subepithelial tumor-like elevation }\end{array}$ \\
\hline Ila & $\begin{array}{l}\cdot \leq 2 \mathrm{~cm} \\
\cdot \text { Steep elevation }\end{array}$ & $\begin{array}{l}>2 \mathrm{~cm} \\
\text { Strong redness } \\
\text { Uneven surface with erosions } \\
\text { Deep depression } \\
\text { Nodular elevation }\end{array}$ \\
\hline Ilb & \multicolumn{2}{|l|}{ Almost all cases are mucosal cancer } \\
\hline \multicolumn{3}{|l|}{ Ilc } \\
\hline Ulceration (-) & $\begin{array}{l}\cdot \leq 2 \mathrm{~cm} \\
\cdot \text { Shallow depression } \\
\cdot \text { Smooth surface } \\
\cdot \text { Minute nodules }\end{array}$ & $\begin{array}{l}>2 \mathrm{~cm} \\
\text { Strong redness } \\
\text { Deep depression } \\
\text { Loss of mucosal surface pattern } \\
\text { Large nodules } \\
\text { Subepithelial tumor-like elevation } \\
\text { Hardness during air inflation }\end{array}$ \\
\hline Ulceration (+) & $\begin{array}{l}\text { - Tapering of a fold tip } \\
\text { - Abrupt cutting of a fold }\end{array}$ & $\begin{array}{l}\text { Clubbing of a fold } \\
\text { Fusion of folds } \\
\text { Hardness during air inflation }\end{array}$ \\
\hline III & \multicolumn{2}{|c|}{ Difficult to estimate the depth of invasion due to accompanying edema } \\
\hline
\end{tabular}




\section{QUANTITATIVE DIAGNOSIS OF EGC}

\section{Depth of invasion}

To predict the depth of invasion in EGCs, the macroscopic morphology of EGCs is mainly considered. For this, it is important to control the amount of inflated air in the stomach during examination and observe the shape of EGCs during peristalsis. ${ }^{18}$ When the surface structure is uniform and there is no ulceration in an elevated lesion, the possibility of mucosal cancer is high. A small depressed lesion without convergence of mucosal folds or dam-like formation is also likely to be a mucosal cancer. In the case of a depressed lesion with convergence of mucosal folds, morphological changes on the tips of converging folds can provide important clues for depth diagnosis (Fig. 4). Fold convergence indicates the presence of fibrosis in the submucosal or deeper layer and does not necessarily mean cancer extension into these layers; many lesions with fold convergence remain as mucosal cancer. ${ }^{18}$ When the tip of the fold is invaded by cancer, a slight initial depression and thinning, then interruption and enlargement, and finally merging and bank formation can appear due to the invasion into deeper layers. Therefore, when the degree of depression is shallow and irregular tapering or abrupt cutting is seen only at the end of the mucosal folds, the possibility of mucosal cancer is high. ${ }^{19}$ On the contrary, a possibility of submucosal cancer increases when the base of the depression is hard, irregular nodules are seen at the margins, or there is clubbing or fusion of the mucosal folds. ${ }^{19}$ When an ulcerative lesion accompanies bank-like elevated margins or fusion of three or more mucosal folds, the possibility of advanced cancer is high.

Abe et al. ${ }^{20}$ proposed a simple scoring model to predict the depth of invasion in differentiated-type EGCs.This scoring model was based on the results of logistic regression analysis which suggested that tumor size $>30 \mathrm{~mm}$, remarkable redness, uneven surface, and margin elevation were significantly associated with deep submucosal invasion. The sensitivity, specificity, and accuracy of this model were $57.3 \%, 86.2 \%$, and $91.1 \%$, respectively. Choi et al. ${ }^{21}$ also reported that the overall accuracy of endoscopy in predicting the depth of invasion in EGCs was 78.0\%, based on the endoscopic criteria of mucosal cancer (smooth surface protrusion or depression, slight marginal elevation, and smooth tapering of the converging folds) and submucosal cancer (irregular surface, marked marginal elevation, and clubbing/abrupt cutting/fusion of the converging folds). Differentiation points to distinguish between mucosal cancer and submucosal cancer according to the macroscopic shape are summarized in Table $1{ }^{22}$ Representative cases of mucosal and submucosal cancers according to the macroscopic shape are presented in Fig. 5.

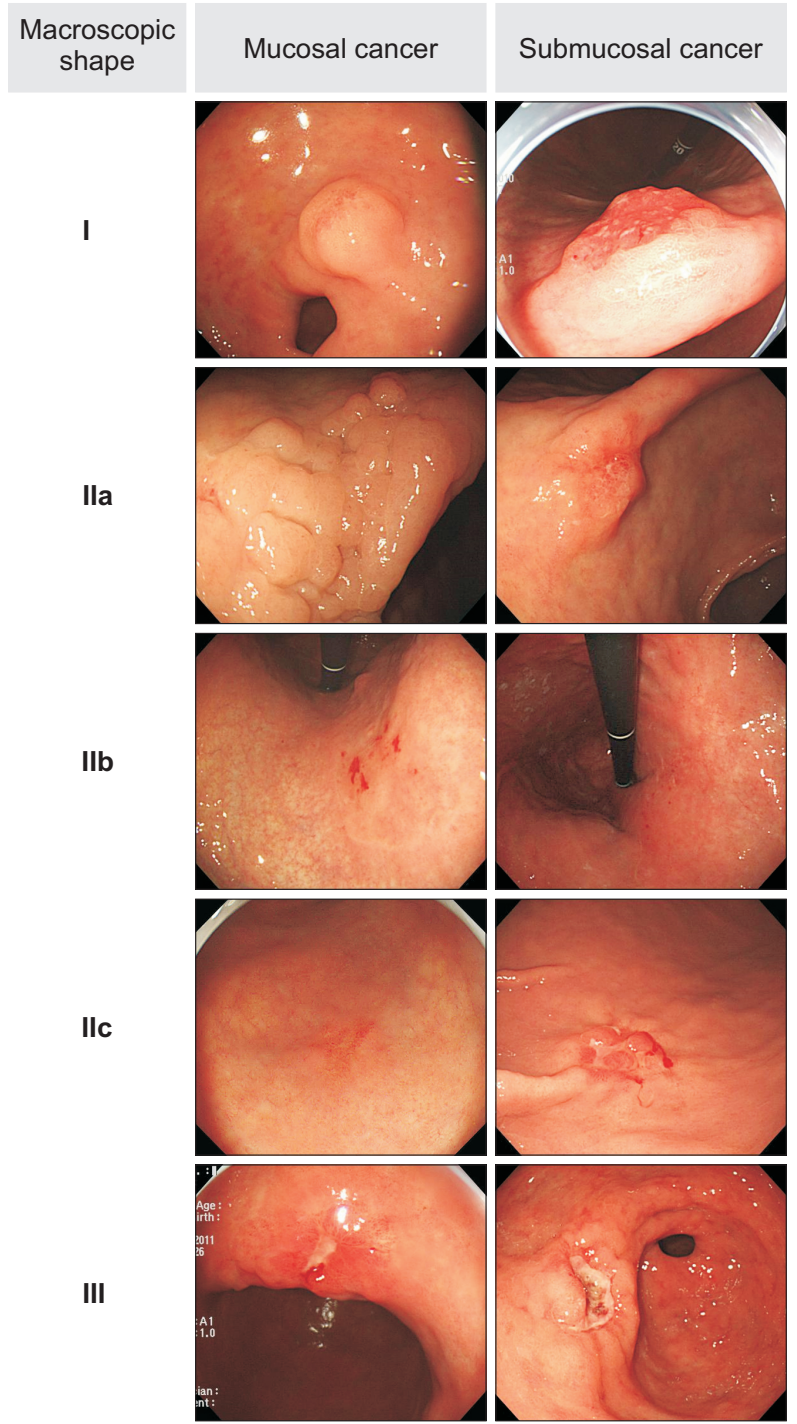

Fig. 5. Representative cases of mucosal and submucosal cancers according to the macroscopic shape.

\section{Horizontal margin delineation}

The horizontal margin of the tumor is determined mainly using conventional endoscopy. ${ }^{23}$ However, when the height and color of the tumor is similar to the surrounding normal mucosa, it is difficult to delineate the horizontal margin accurately. In a Japanese study, the horizontal margin was unclear in $18.9 \%$ of EGC cases during conventional endoscopy, and the characteristic endoscopic findings of these cases showed type 0-IIb lesion. ${ }^{24}$

Indigo carmine chromoendoscopy, which enables the visualization of the irregularity of the mucosal structure clearly, has been used for over 40 years, and it is still a useful modality for identifying gastric lesions. ${ }^{25}$ However, it is difficult to accurately identify the horizontal margins of the tumor in the case of marked intestinal metaplasia because the indigo carmine simply enhances the irregularity of the tumor surface. ${ }^{26}$ Acetic acid chromoendoscopy was 

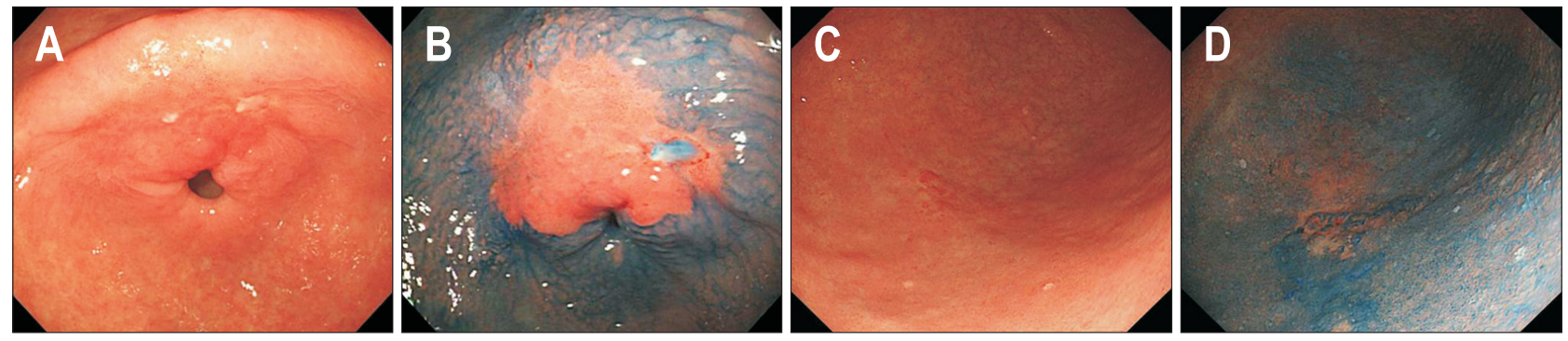

Fig. 6. Acetic acid-indigo carmine (Al) chromoendoscopy. (A) A slightly reddish flat lesion is seen at the lesser curvature of the prepylorus. (B) After Al chromoendoscopy, the lesion's borders become distinct and the clarity of the image is high. This lesion was finally diagnosed as well-differentiated tubular adenocarcinoma after endoscopic submucosal dissection. (C) A discolored depressed lesion is seen at the greater curvature of the lower body. (D) After Al chromoendoscopy, the lesion's border is still indistinct and the image is mottled. This lesion was finally diagnosed as signet ring cell carcinoma after distal gastrectomy.

first used to observe the columnar epithelium of Barrett's esophagus $^{27}$ and has since been used in gastric lesions. The transient whitening of the epithelium after acetic acid spraying is caused by an increase in opacity, which corresponds to the reversible alternation of the three-dimensional structures of the cytoplasmic proteins. ${ }^{28}$ However, the usefulness of acetic acid chromoendoscopy is reported only in about $42 \%$ to $53 \%$ of EGCs. ${ }^{28}$ Acetic acid-indigo carmine (AI) chromoendoscopy, which spays acetic acid and indigo carmine sequentially, is a chromoendoscopy method that combines the advantages of acetic acid and indigo carmine chromoendoscopy in the delineation of the horizontal margins of EGCs. ${ }^{26}$ Using the AI chromoendoscopy, the horizontal margins of differentiated-type EGCs, and not undifferentiated-type EGCs, can be observed more clearly (Fig. 6). ${ }^{29}$ Therefore, if the endoscopic biopsy shows the undifferentiated type, biopsy samples must be collected from the apparently non-cancerous mucosa around the lesion before ESD, and the resection margins should be determined after histopathological confirmation of the absence of cancerous invasion. ${ }^{23}$

\section{CONCLUSIONS}

The detection of EGCs is increasing as screening endoscopy is being commonly performed. Furthermore, with the development of imaging technology, the detection of smaller and non-typical lesions is also increasing. However, the discovery of such lesions still requires careful attention from endoscopists and an endoscopic knowledge of EGCs. In particular, the paradigm shift from surgical resection to ESD in the treatment of EGCs is progressing. Advanced diagnostic modalities, such as endosonography or magnifying endoscopy, are helpful for qualitative and quantitative diagnosis of EGCs. However, there is a limitation in that these modalities are not available in most hospitals. There- fore, it is crucial to evaluate EGCs systematically during conventional endoscopy for successful treatment.

\section{CONFLICTS OF INTEREST}

G.H.K. is an editorial board member of the Journal but was not involved in the peer reviewer selection, evaluation, or decision process of this article. No other potential conflicts of interest relevant to this article were reported.

\section{ACKNOWLEDGEMENTS}

This study was supported by the Medical Research Center Program through the National Research Foundation of Korea grant funded by the Korea government (NRF2015R1A5A2009656).

\section{ORCID}

Gwang Ha Kim https://orcid.org/0000-0001-9721-5734

\section{REFERENCES}

1. Jun JK, Choi KS, Lee HY, et al. Effectiveness of the Korean National Cancer Screening Program in reducing gastric cancer mortality. Gastroenterology 2017;152:1319-1328.

2. Hamashima C; Systematic Review Group and Guideline Development Group for Gastric Cancer Screening Guidelines. Update version of the Japanese Guidelines for Gastric Cancer Screening. Jpn J Clin Oncol 2018;48:673-683.

3. Soetikno R, Kaltenbach T, Yeh R, Gotoda T. Endoscopic mucosal resection for early cancers of the upper gastrointestinal tract. J Clin Oncol 2005;23:4490-4498. 
4. Gotoda T. Endoscopic resection of early gastric cancer. Gastric Cancer 2007;10:1-11.

5. Choi MK, Kim GH, Park DY, et al. Long-term outcomes of endoscopic submucosal dissection for early gastric cancer: a single-center experience. Surg Endosc 2013;27:4250-4258.

6. Jeon HK, Kim GH, Lee BE, et al. Long-term outcome of endoscopic submucosal dissection is comparable to that of surgery for early gastric cancer: a propensity-matched analysis. Gastric Cancer 2018;21:133-143.

7. Gotoda T, Yanagisawa A, Sasako M, et al. Incidence of lymph node metastasis from early gastric cancer: estimation with a large number of cases at two large centers. Gastric Cancer 2000;3:219-225.

8. Yao K. The endoscopic diagnosis of early gastric cancer. Ann Gastroenterol 2013;26:11-22.

9. The Paris endoscopic classification of superficial neoplastic lesions: esophagus, stomach, and colon. November 30 to December 1, 2002. Gastrointest Endosc 2003;58(6 Suppl):S3S43.

10. Endoscopic Classification Review Group. Update on the Paris classification of superficial neoplastic lesions in the digestive tract. Endoscopy 2005;37:570-578.

11. Suzuki S, Suzuki H, Endo M, Takemoto T, Kondo T. Endoscopic diagnosis of early cancer and intestinal metaplasia of the stomach by dyeing. Int Surg 1973;58:639-642.

12. Noh CK, Jung MW, Shin SJ, et al. Analysis of endoscopic features for histologic discrepancies between biopsy and endoscopic submucosal dissection in gastric neoplasms: 10year results. Dig Liver Dis 2019;51:79-85.

13. Kim Y, Yoon HJ, Kim JH, et al. Effect of histologic differences between biopsy and final resection on treatment outcomes in early gastric cancer. Surg Endosc 2020;34:5046-5054.

14. Kim GH, Kim KB, Lim EK, et al. Analysis of endoscopic electronic image of intramucosal gastric carcinoma using a software program for calculating hemoglobin index. J Korean Med Sci 2006;21:1041-1047.

15. Honmyo U, Misumi A, Murakami A, et al. Mechanisms producing color change in flat early gastric cancers. Endoscopy 1997;29:366-371.

16. Adachi Y, Mori M, Enjoji M, Sugimachi K. Microvascular architecture of early gastric carcinoma. Microvascular-histopathologic correlates. Cancer 1993;72:32-36.

17. Yao K, Yao T, Matsui T, Iwashita A, Oishi T. Hemoglobin content in intramucosal gastric carcinoma as a marker of histologic differentiation: a clinical application of quantitative electronic endoscopy. Gastrointest Endosc 2000;52:241-
245.

18. Sano T, Okuyama Y, Kobori O, Shimizu T, Morioka Y. Early gastric cancer: endoscopic diagnosis of depth of invasion. Dig Dis Sci 1990;35:1340-1344.

19. Ono H, Yoshida S. Endoscopic diagnosis of the depth of cancer invasion for gastric cancer. Stomach Intest 2001;36:334340.

20. Abe S, Oda I, Shimazu T, et al. Depth-predicting score for differentiated early gastric cancer. Gastric Cancer 2011;14:35-40.

21. Choi J, Kim SG, Im JP, Kim JS, Jung HC, Song IS. Endoscopic prediction of tumor invasion depth in early gastric cancer. Gastrointest Endosc 2011;73:917-927.

22. Sumiyama K. Past and current trends in endoscopic diagnosis for early stage gastric cancer in Japan. Gastric Cancer 2017;20(Suppl 1):20-27.

23. Yao K, Nagahama T, Matsui T, Iwashita A. Detection and characterization of early gastric cancer for curative endoscopic submucosal dissection. Dig Endosc 2013;25 Suppl 1:44-54.

24. Nagahama T, Yao K, Maki S, et al. Usefulness of magnifying endoscopy with narrow-band imaging for determining the horizontal extent of early gastric cancer when there is an unclear margin by chromoendoscopy (with video). Gastrointest Endosc 2011;74:1259-1267.

25. Okabayashi T, Gotoda T, Kondo H, et al. Usefulness of indigo carmine chromoendoscopy and endoscopic clipping for accurate preoperative assessment of proximal gastric cancer. Endoscopy 2000;32:S62.

26. Yamashita H, Kitayama J, Ishigami H, et al. Endoscopic instillation of indigo carmine dye with acetic acid enables the visualization of distinct margin of superficial gastric lesion: usefulness in endoscopic treatment and diagnosis of gastric cancer. Dig Liver Dis 2007;39:389-391.

27. Guelrud M, Herrera I. Acetic acid improves identification of remnant islands of Barrett's epithelium after endoscopic therapy. Gastrointest Endosc 1998;47:512-515.

28. Sakai Y, Eto R, Kasanuki J, et al. Chromoendoscopy with indigo carmine dye added to acetic acid in the diagnosis of gastric neoplasia: a prospective comparative study. Gastrointest Endosc 2008;68:635-641.

29. Lee BE, Kim GH, Park DY, et al. Acetic acid-indigo carmine chromoendoscopy for delineating early gastric cancers: its usefulness according to histological type. BMC Gastroenterol 2010;10:97. 